\title{
EDITORIAL
}

\section{The prognosis impact of BCR-ABL P-loop mutations: worse or not worse?}

Leukemia (2007) 21, 193-194. doi:10.1038/sj.leu.2404490

In a paper recently published in Leukaemia, Jabbour et al. ${ }^{1}$ report an original retrospective monocentric study of chronic myelogenous leukemia $(\mathrm{CML})$ patients resistant to imatinib who were genotyped for the presence of $\mathrm{ABL}$ kinase domain mutations. Sixty-two out of 171 resistant patients (36\%) harboured 66 BCR-ABL mutations, with 24 P-loop, three T315I and 39 mutations falling in other sites of the ABL kinase domain. A multivariate analysis performed on the whole cohort (resistant patients with and without mutation) determined some poor prognosis features (older age, prior interferon therapy, advanced phase at the time of imatinib failure, clonal evolution and high peripheral basophil counts at resistance). The identification of a mutation, considering the mutation as a time-dependent covariate, was associated with worse survival, measured from the time of imatinib initiation. Strikingly, the analysis of survival demonstrated that the mutation status had no impact on survival and that P-loop mutations were not associated with a poor outcome as another preliminary study (Hochhaus A, Ernst et al., Blood 2005; 106: 316a, abstract no. 1086). We previously reported this year ${ }^{2}$ as others, ${ }^{3,4}$ in a multicentric retrospective study, 89 patients all harbouring a BCR-ABL mutation and we demonstrated that P-loop and T315I mutations selectively impaired the outcome of imatinib-resistant CML patients, in contrast to other mutations. The observation period between these two studies was the same (1999-2005), as well as the methods for mutation detection (polymerase chain reaction (PCR) and sequencing), the median follow-up (38 months for MD Anderson, 39 months for $\mathrm{Fi}(\varphi)$-LMC) and the imatinib dose at the initiation of this treatment $(90 \%$ $400 \mathrm{mg} \mathrm{day}^{-1}$ in Texas, 95\% in France). However, we identified a number of differences that might be responsible for the different conclusions drawn in these two articles. We concentrated on the population of patients of MD Anderson harbouring a BCR-ABL mutation, in order to be able to compare the results with our own cohort (where all patients harboured a mutation) and try to identify the variables involved in the different results obtained. It is of note that in Jabbour et al. ${ }^{1}$ the P-loop definition included amino acids 244-255, instead of 248-255 in our study (i.e. excluding the 244 mutations); finally 24 patients with P-loop mutations were studied in MD Anderson and 24 in our study. The median age at CML diagnosis was higher in MD Anderson (57 vs 51.7). A significant subset of patients in chronic phase received interferon- $\alpha$ in France $(72 / 87$ (83\%)) whereas a smaller proportion of the patients did in Texas (43/62 (69\%)). We have demonstrated in our study, that, for chronic phase, exposure to interferon- $\alpha$ was significantly associated with longer survival in a multivariate analysis. The median overall survival since the time of mutation detection in Jabbour et al. ${ }^{1}$ was $\sim 19$ months for non-P-loop mutations (See Figure 1 of this paper ${ }^{1}$ ) and not reached for P-loop mutations whereas it was 18 months for P-loop and not reached for other mutations (excluding T315I) in our study and $89 \%$ of the latter received interferon- $\alpha$ (see Table 1$) ;^{2}$ therefore, we suspect that this variable might be involved in the differences observed in survival. In addition, at the time of discovery of the mutation, more patients were in advanced phase in Texas (30 in accelerated phase +10 in blastic phase out of 62 patients (65\%), vs 21 and 11 out of 89 patients (36\%) in France) and it is difficult to understand why there are so few T315I (3/62 patients) mutations identified in the American study (18/89 in the French study), because these mutations are preferentially observed in the advanced phases of the disease (as in the American series) and are of very poor prognosis. $^{2}$ In the American study, the T315I patients have been included in the cohort 'non-P-loop mutations' for analysis and might unfavourably affect the survival of this cohort.

Additionally, in the American study patients were not censored at allogeneic stem cell transplantation, secondgeneration tyrosine kinase inhibitors (TKIs) or other investigational drugs whereas in our study they were all censored for any of these variables. As raised by Jabbour et al. ${ }^{1}$ in Table 4, the proportion of patients treated with second-generation TKIs is larger in the cohorts of mutated patients $(46 \%$ of the patients in the group $\mathrm{P}$-loop, $42 \%$ in the group of other mutations), however, we speculate that the three T315I mutated patients (over 16) have not responded and should worsen the survival curve of this group, and the relative proportion of patients treated with either dasatinib or nilotinib is unknown. As the sensitivity of BCR-ABL mutated CML patients to new TKIs might be different, it would be interesting to know the proportion of the two TKI-treated patients in each P-loop and in other mutation groups.

We re-analysed our data since the time of imatinib initiation (instead of the time of detection of mutation as previously ${ }^{2}$ ) and took the same amino-acid residues to redefine our P-loop group (i.e. we thus included in the P-loop nine M244V patients who were subtracted from the group 'other' mutations) as Jabbour et al. did, and drew overall survival curves for all phases of the

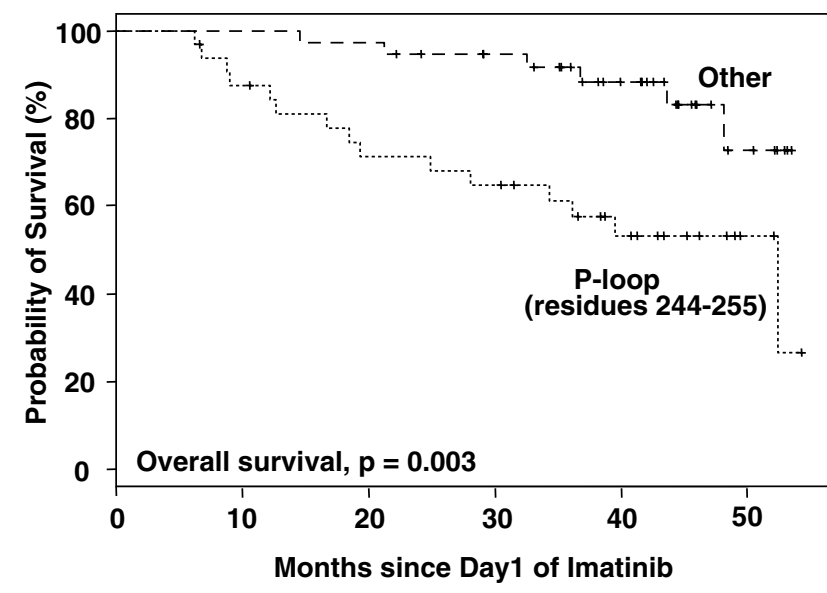

Figure 1 Overall survival of all the CML patients in our series harbouring P-loop (redefined as between residues 244-255; dotted line) or other BCR-ABL mutations (which were subtracted of the M244V mutants; dashed line) excluding T315I, analysed since the initiation of imatinib, according to Kaplan-Meier method. 
disease, excluding the T315I mutations, in order to compare the French and the American cohorts. As shown in Figure 1 here, the Kaplan-Meier overall survival curves of newly defined P-loop mutated patients and other mutated patients (excluding T315I and now M244V) are nearly the opposite of that in the American paper (Figure 2c in Jabbour et al. ${ }^{1}$ ), with a significantly poorer outcome for P-loop mutations $(P=0.003)$.

Despite the fact that somewhat different statistical methodologies have been used for the analysis in these two studies, we believe that the important differences noted illustrate the critical importance of patient selection and of a monocentric effect on the results, and probably, at various levels, the impact of new agents and stem cell transplantation. In addition, the genetic background of the CML patient populations between the USA and France might differ and could affect the response to imatinib and the onset of BCR-ABL mutations. However, it is likely that imatinib resistance in these patients harbouring $B C R-A B L$ mutations, particularly in advanced phase patients, is multifactorial. A meta-analysis of the data between the two cohorts seems mandatory to try to elucidate the differences in survival observed.

\footnotetext{
FE Nicolini ${ }^{1}$, S Corm $^{2}$, Q-H Lê ${ }^{1}, C$ Roche-Lestienne ${ }^{3}$ and C Preudhomme ${ }^{1,3}$ ${ }^{1}$ Hematology Department, E. Herriot Hospital, Lyon, France;
}

${ }^{2}$ Hematology Department, CHRU, Lille, France and ${ }^{3}$ INSERM U 817, Institut de Recherche contre le Cancer, Lille, France

E-mail: cpreudhomme@chru-lille.fr

\section{References}

1 Jabbour E, Kantarjian $\mathrm{H}$, Jones D, Talpaz M, Bekele N, O'Brien S et al. Frequency and clinical significance of BCR-ABL mutations in patients with chronic myeloid leukemia treated with imatinib mesylate. Leukemia 2006; 20: 1767-1773.

2 Nicolini FE, Corm S, Lê Q-H, Sorel N, Hayette S, Bories D et al. Mutation status of 89 imatinib mesylate resistant chronic myelogenous leukemia patients and clinical outcome. A retrospective analysis from the french intergroup of $\mathrm{CML}(\mathrm{Fi}(\varphi)$-LMC group). Leukemia 2006; 20: 1061-1066.

3 Branford S, Rudzki Z, Walsh S, Parkinson I, Grigg A, Szer J et al. Detection of BCR-ABL in patients with CML treated with imatinib is virtually always accompanied by clinical resistance, and mutations in the ATP-binding loop (P-loop) are associated with a poor prognosis. Blood 2003; 102: 276-283.

4 Soverini S, Martinelli G, Rosti G, Bassi S, Amabile M, Poerio A et al. $\mathrm{ABL}$ mutations in late chronic phase chronic myeloid leukaemia patients with up-front cytogenetic resistance to imatinib are associated with a greater likelihood of progression to blast crisis and shorter survival: a study from the GIMEMA working party on chronic myeloid leukaemia. J Clin Oncol 2005; 23: 4100-4109. 